\title{
Observational properties of magnetic white dwarfs
}

\author{
Lilia Ferrario* \\ Mathematical Sciences Institute, The Australian National University, \\ Canberra, ACT 2601, Australia \\ *E-mail: Lilia.Ferrario@anu.edu.au \\ www.maths.anu.edu.au/people/lilia-ferrario
}

\begin{abstract}
There are no known examples of magnetic white dwarfs with fields larger than $\sim 3 \mathrm{MG}$ paired with a non-degenerate companion in detached binary systems. The suggestion is that highly magnetic, isolated white dwarfs may originate from stars that coalesce during common envelope evolution while those stars that emerge from a common envelope on a close orbit may evolve into double degenerate systems consisting of two white dwarfs, one or both magnetic.

The presence of planets or planetary debris around white dwarfs is also a new and exciting area of research that may give us important clues on the formation of first and second generation planetary systems, since these place unique signatures in the spectra of white dwarfs.
\end{abstract}

Keywords: White dwarfs, magnetic fields, double degenerates, planets

\section{Introduction}

Grw $+70^{\circ} 8247 \frac{1}{\underline{1}}$ was the first magnetic white dwarf (MWD) discovered. This object exhibits a strongly circularly polarised spectrum that is nearly featureless except for broad shallow features that became known as "Minkowski bands". Computations of $\mathrm{H}$ transitions in strong magnetic fields in the mid-80s revealed that these features are Zeeman shifted $\mathrm{H}$ lines in a field of $100-320 \mathrm{MG}^{2}$. From just a handful of objects that were known in the early 80s, the number has increased to $\sim 300$ isolated MWDs and $\sim 170$ MWDs in binaries $\underline{3}$.

The modelling of Zeeman spectral lines is the best way to determine the field strength and structure of isolated MWDs. The detection of cyclotron lines in the optical to IR bands offers an additional method for the study of MWD fields in interacting binaries $\underline{\underline{4}} \underline{\underline{\underline{7}}}$. Whilst research on these enigmatic objects has led to a better understanding of stellar magnetism, it has also raised a number of unanswered questions regarding the pre-MWD evolution and the origin and structure of stellar magnetic fields.

In this brief review I will report the latest thoughts on the origin and evolution of MWDs and I will highlight their importance as possible progenitors of supernova events, millisecond pulsars, and as hosts of first and second generation planets. More comprehensive reviews on all aspects of this subject are available in the literature 3.8 .

\section{Origin of magnetic fields in white dwarfs}

According to the fossil field scenario, magnetic fields present in the interstellar medium (ISM) at the time of star formation freeze into the radiative regions of protostars. Fluctuations in the ISM field strength would explain the field spread observed 
in main sequence (MS) stars. In this picture the magnetic flux is then conserved as the star evolves off the MS to the compact star phase. Since the peculiar Ap/Bp stars are known to have large-scale ordered fields, these stars have been proposed to be the progenitors of the MWDs $\stackrel{9}{ }$. However, one should expect that MWDs ought to occur as often in detached binaries as in single stars. The Sloan Digital Sky Survey has identified thousands of WD+M dwarfs in detached spectroscopic binaries $\frac{10}{}$. The peculiarity is that none of these systems harbours a MWD. The sample of WDs within $20 \mathrm{pc}$ has revealed that $19.6 \pm 4.5 \%$ of them have MS companions $\frac{11}{1}$. The magnitude limited Palomar Green (PG) Survey has shown that $23-29 \%$ of hot WDs have cool companions $\frac{12}{2}$. Thus, the current MWD sample of about 300 stars should contain at least $40-90$ objects with a non-degenerate companion $\stackrel{13,14}{\underline{n}}$, but instead it does not include any. This incongruity suggests that the origin of magnetic fields in WDs may be linked to their duplicity $\underset{15}{\underline{15}}$. Spiralling stellar cores during common envelope (CE) evolution give rise to differential rotation. Differential rotation and a seed poloidal magnetic field in the envelope of the primary star would produce strong toroidal and poloidal fields which would stabilise each other and limit field growth. The final poloidal field strength has been found to be proportional to the initial amount of differential rotation, but independent of the initial seed field $\frac{16}{6}$ (see left panel of Fig. 1).

MWDs in non-interacting double degenerate systems (DDs) and in the magnetic cataclysmic variables could form via a similar mechanism during CE evolution. The closer the stellar cores get before the expulsion of the envelope, the stronger the magnetic field of the proto-MWD emerging from the CE will be $\frac{15}{}$.

It has been known since $1988 \frac{17}{17}$ that high field MWDs have masses that are on average higher than those of their non-magnetic or weakly magnetic counterparts $(\sim 0.78 M \odot \underline{3}$ versus $\sim 0.66 M \odot \stackrel{18}{18})$. Binary population synthesis calculations are consistent with the scenario that MWDs arise from stars that merge during $\mathrm{CE}$ evolution $\underline{19}$ and predict that MWDs formed through this channel are on average more massive than non-magnetic white dwarfs, as required by observations (see right panel of Fig. (1).

In an alternative scenario, seed fields in the accretion disc formed by the debris of a low-mass star tidally disrupted by its companion during CE, are augmented by turbulence and shear and transferred on to what will become a MWD 20 . 3-D hydrodynamic calculations of merging double WDs have also been carried out $\underline{21}$. These studies show that a differentially rotating, hot, convective corona forming around the more massive star during the merging process produces strong magnetic fields (see left panel of Fig. 2). Population synthesis computations of double WD mergers appear to be consistent with observations (see right panel of Fig. 22).

On the other hand one should not completely dismiss the fossil field scenario for the formation of $\mathrm{MWDs}^{9} \underline{22}$, since it is feasible that MWDs could exist in binaries although hidden in the glare of a brighter non-degenerate companion $\underline{23}$ (Sirius-type systems). Thus, the lack of MWD+M dwarfs in detached binaries could perhaps 

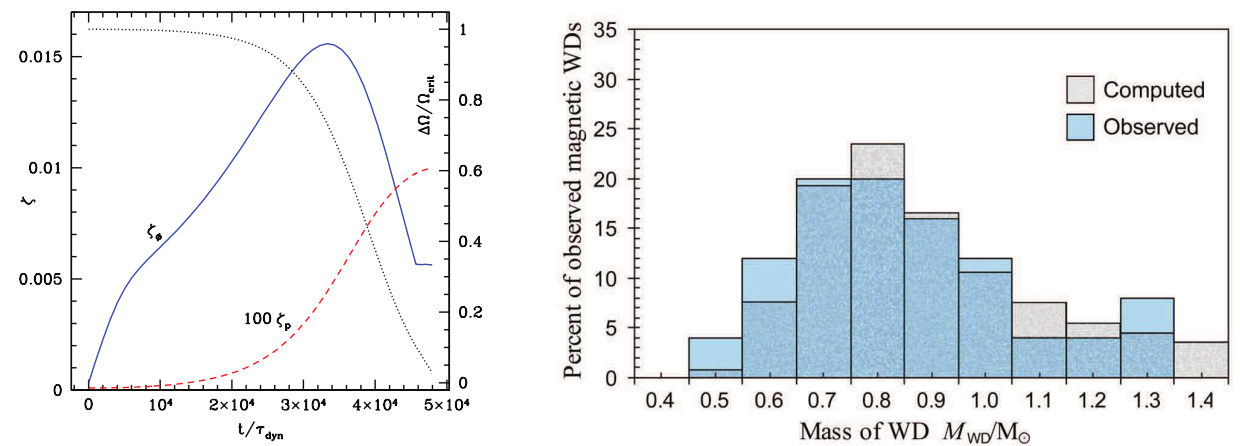

Fig. 1. Left panel: Evolution of $B_{\mathrm{p}}$ (poloidal field) and $B_{\phi}$ (toroidal field, $\zeta=\sqrt{\eta} \approx B$ ) during a star merging event is shown on the left hand axis. Here, $B_{\phi}$ reaches a peak and then decays till equilibrium with the poloidal field is reached. The decay of differential rotation $\Delta \Omega$ is shown on the right hand axis 16 . Right panel: Mass distribution of observed MWDs (blue histogram) compared with the computed sample (grey histogram) $\underline{19}$. The most common predicted progenitors are Asymptotic Giant Branch (AGB)/MS star (61\%), AGB/M-dwarf (17\%) and RGB/WD (14\%) mergers. Other routes comprise less than 1 per cent of the total.
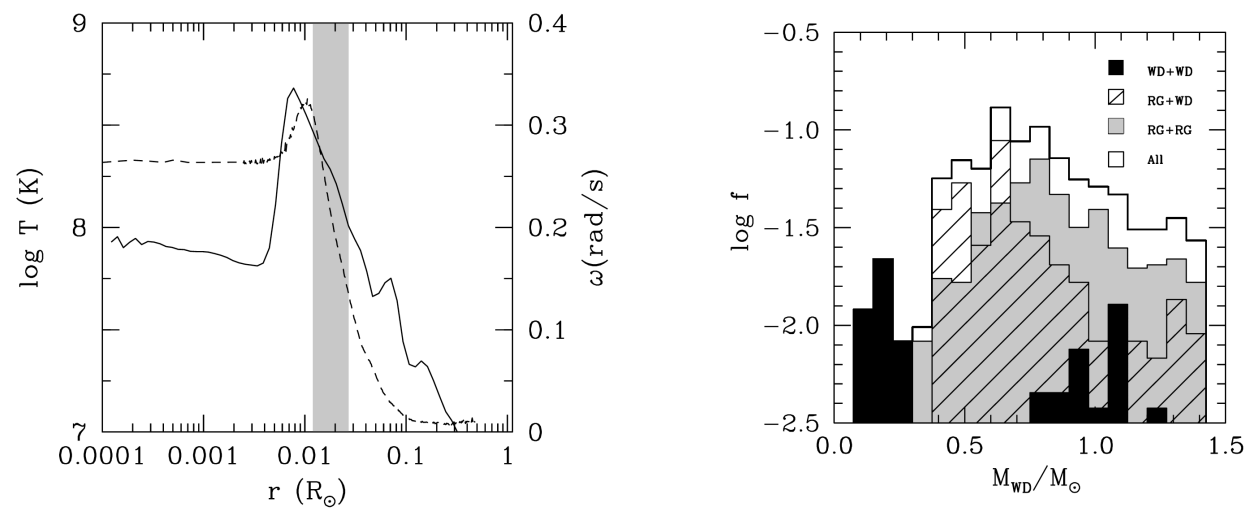

Fig. 2. Left panel: Dynamo configuration in the merger of two stars of $0.6 \mathrm{M}_{\odot}$ and $0.8 \mathrm{M}_{\odot}$. The temperature profile (solid line) is shown on the left hand axis and the rotational velocity (dashed line) on the right axis as a function of the radius of the resulting WD. Right panel: Mass frequency of merger channels 21 . Black histogram: white dwarf-white dwarf mergers; dashed histogram: red giant-white dwarf mergers; shaded histogram: red giant-red giant mergers. The solid line represents the total mass distribution.

be attributed to the manner in which magnetic Ap/Bp stars (the progenitors of the MWDs in the fossil field hypothesis) are paired with their secondary stars. If a sufficient number of Sirius-type systems hosting a MWD is discovered, this finding could support the view that there is more than one formation channel for MWDs. In this context, I wish to draw attention to the atmospheric modelling of cool MWDs $\frac{24}{2}$ showing that the spectral characteristics of 10 out of the 16 objects 
under consideration in this study can be best modelled as unresolved magnetic DDs (see section 3) whose progenitors could indeed have been (magnetic) Sirius-type systems.

\section{Double degenerate systems}

Type Ia supernovae (SNeIa), which are used as standard candles to determine the expansion history of the universe, are caused by accreting white dwarfs which undergo thermonuclear explosions. Since different SNIa progenitors may lead to different peak luminosities or different light-curve width-luminosity relations, their characteristics as a function of redshift could imitate the behaviour of a time-varying dark energy equation of $\operatorname{state}^{25}$. Since we still know very little about the origin of SNeIa, this is known as the "SNIa progenitor puzzle".

The thermonuclear disruption of a WD could occur through the merger of two WDs (also called the DD route). On the other hand, a DD merger may also lead to the formation of a millisecond pulsar via accretion induced collapse 26.27 . If one of the two WDs has a strong magnetic field, a collapse could lead to the formation of a high field pulsar or a magnetar 28,29 . What causes a collapse rather than an explosion is still being strongly debated. The presence of an intense magnetic field has only very recently been included in simulations of the post-merger evolution of DDs ${ }^{30}$. A list of the currently known magnetic DDs is given in Table 1 below.

LB 11146 was identified in $1993^{31}$ as a DD binary consisting of a non-magnetic and a strongly magnetic $(\sim 670 \mathrm{MG})$ WD. The WD masses were estimated to be about $0.9 M_{\odot}$ and thus, LB 11146 was considered to be a potential SNIa progenitor. However, further observations of LB $11146^{32}$ revealed the presence of a long orbital period ( $\sim 130$ days) and a separation of $\sim 0.6$ AU. Since the size of the orbit is smaller than the size of its AGB progenitor, this indicated that the two stars went through at least one CE phase. The long orbital period of LB 11146 is at odds with those of most other known DDs 32 , whose periods vary from a few hours to a few days 33 .

Another remarkable DD is NLTT 12758, whose MWD component has a polar field of $\sim 3 \mathrm{MG}$. This DD exhibits variations at the rotation (spin) period of the MWD $(\sim 22$ mins $)$ and at the orbital period $(\sim 11 \mathrm{hrs}) \stackrel{34,35}{ }$. The best fit model gives masses of $\sim 0.9$ and $\sim 1 \mathrm{M}_{\odot}$ for the non-magnetic and magnetic components respectively. As for LB 11146, the total mass exceeds the Chandrasekhar's limiting mass, so in principle this DD could be a potential SNIa progenitor. However, its orbital parameters also place this DD on a merging timescale comparable to the Hubble time 35 .

Follow-up observations of EUVE J1439+750 ${ }^{36}$, G 183-35 $\stackrel{37}{ }$, and G 141-2 ${ }^{37}$ have shown that there is no evidence for variability in any of these objects, at least within the given observational limitations. More observations are needed to establish the orbital parameters of the remaining magnetic DDs.

According to the merger scenario for magnetic field formation 15 , in non- 
Table 1. Currently known magnetic DDs

\begin{tabular}{llll}
\hline Name & Other name & Variability/Periods & Notes \& References \\
\hline $0040+000$ & SDSS J004248.19+001955.3 & $\ldots$ & 38 \\
$0121-429$ & LHS 1243 & $\ldots$ & 24 \\
$0239+109$ & G 4-34, LTT 0886 & $\ldots$ & 39 \\
$0325-857$ & RE J0317-853, EUVE J0317-855 & $P_{\text {spin }} \sim 725 \mathrm{~s}, P_{\text {orb }} \sim 2092$ yrs & $40,41,42$ \\
$0410-114$ & G160-51, NLTT 12758 & $P_{\text {spin }} \sim 23$ min, $P_{\text {orb }} \sim 0.6 \mathrm{~d}$ & $34,24,35$ \\
& SDSS J092646.88+132134.5 & $\ldots$ & $\mathrm{CPM}, 43$ \\
$0512+284$ & LSPM J0515+2839 & $\ldots$ & 24 \\
$0745+303$ & SDSS J074853.07+302543.5 & $\ldots$ & $\mathrm{CPM}, 44$ \\
& SDSS J092646.88+132134.5 & $\ldots$ & $\mathrm{CPM}, 43$ \\
$0945+246$ & LB 11146 & $P_{\text {orb }} \sim 130 \mathrm{~d}$ & 32 \\
$1026+117$ & LHS 2273 & $P_{\text {spin? }} \sim 35-45$ min & 37,24 \\
$1300+590$ & SDSS J130033.48+590407.0 & $\ldots$ & $\mathrm{CPM}, 45$ \\
$1330+015$ & G62-46 & $\ldots$ & 24 \\
$1440+753$ & EUVE J1439+750 & $\ldots$ & 46 \\
$1503-070$ & GD 175 & $\ldots$ & 24 \\
& SDSS J150746.80+520958.0 & $\ldots$ & $\mathrm{CPM}, 43$ \\
$1506+399$ & CBS 229, SDSS J150813+394504 & $\ldots$ & $\mathrm{CPM}, 44$ \\
$1514+282$ & SDSS J1516+2803 & $\ldots$ & 24 \\
$1713+393$ & NLTT 44447 & $\ldots$ & 24 \\
$1814+248$ & G 183-35 & 50 min-yrs? & Double MWDs, $47,37,24$ \\
$1818+126$ & G 141-2 & yrs? & $37,48,24$ \\
\hline
\end{tabular}

interacting WD-MWD binaries that have gone through CE evolution, the nonmagnetic WD would form first following a $\mathrm{CE}$ phase that would bring the two stars closer to each other. The absence of a magnetic field implies that there must be additional and as yet unknown conditions that need to be satisfied for a magnetic field to be generated during a CE (e.g. stellar separation, mass ratio). It is during a subsequent $\mathrm{CE}$ phase that the companion star would develop a magnetic field and would evolve into a MWD. Those DDs with large binary separations (e.g. common proper motion pairs) that did not undergo $\mathrm{CE}$ evolution would be the results of triple systems where the MWD formed via a CE merger. There is at least one DD, G 183-35, where both WDs are magnetic. This system could have formed following two CE phases each producing a MWD. If this is the case, the implication is that the components of G 183-35 form a close binary system and that further observations may be needed to search for very short orbital periods.

Finally, one needs to address the question why not all DDs, particularly those at small binary separations, harbour at least one MWD. The answer could be that either (i) the WD precursor entering CE evolution did not already have a degenerate core $\frac{19}{}$; or (ii) convective nuclear burning in the core of the WD precursor took place after it emerged from CE evolution, thus destroying any generated frozen-in magnetic field $\underline{19}$ or, as already mentioned above, (iii) there are additional factors that come into play for the generation of a magnetic field when two stars enter a CE phase.

According to the alternative fossil field hypothesis, magnetic DDs would be 
the descendants of Sirius-type systems ${ }^{23}$ where the MWD in the binary would the progeny of a magnetic Ap/Bp star.

\section{Planets around MWDs}

The accretion of planetary debris by WDs could explain the existence of DZ WDs $\underline{49}$ which are characterised by strong metal lines (e.g. Ca, $\mathrm{Si}, \mathrm{Mg}, \mathrm{Na}$ and sometimes Fe, $\mathrm{Ti}$ and $\mathrm{Cr}$ ) in their spectra (see Fig. 3). Interestingly, the incidence of magnetism
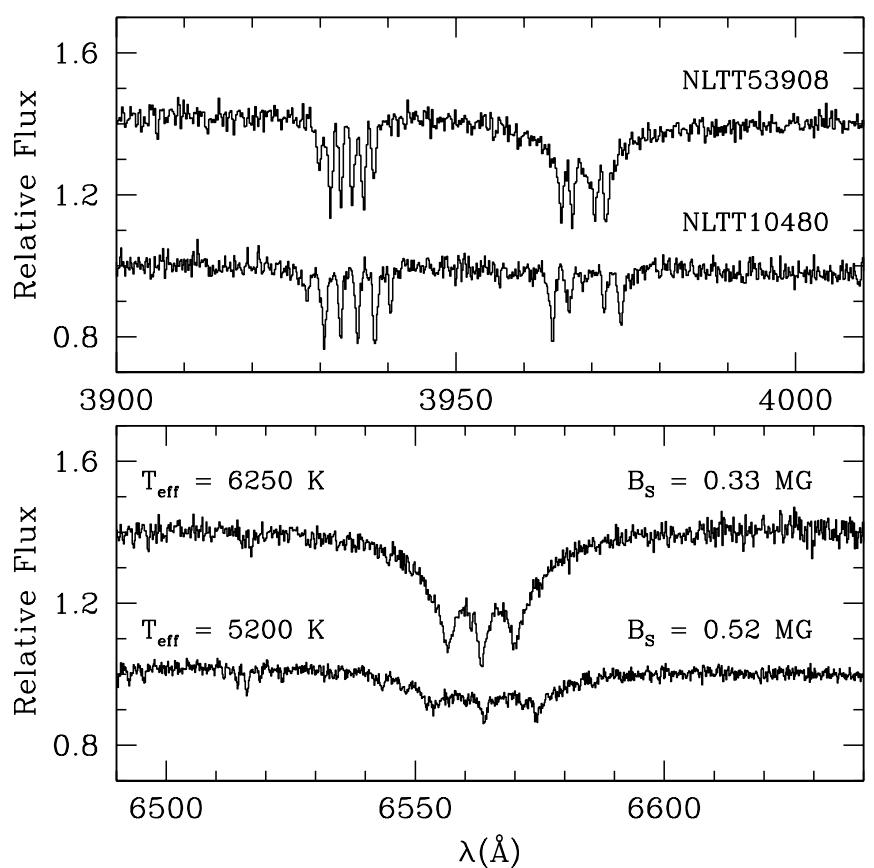

Fig. 3. Linear Zeeman splitting of Ca II (H and K) and H $\alpha$ in NLTT $10480 \stackrel{50}{5}$ and NLTT 5390851.

among cool $(<9,000 \mathrm{~K})$ DZ WDs is about $13 \%$, which is considerably higher than among the general population of WDs $\frac{51,52}{}$ (magnitude-limited samples indicate that only $\sim 2-5 \%$ are magnetic $\left.{ }^{53}\right)$. In order to explain this peculiarity, it has been suggested that the progenitor AGB star underwent a CE phase with a giant gaseous planet, which led to the formation of a MWD $\stackrel{54}{ }$. The atmosphere of this MWD would then become polluted due to the accretion of rocky planets and/or asteroid rubble 55,56 . The CE formation channel for DZ MWDs could be strengthened if the incidence of magnetism among DC (featureless) WDs, which belong to the same stellar population as the DZ's 55 , were found to be considerably different. In this picture, the MS progenitors of DZ MWDs could have been intermediate mass stars that had planetary systems composed by Earth-like planets and at least one close-in 
gaseous giant responsible for the WD's magnetic field $\stackrel{55}{ }$.

It is still rather unclear what the incidence of magnetism among DZ WDs with $T_{\text {eff }} \geq 10,000 \mathrm{~K}$ is $\frac{51}{}$, although preliminary studies seem to indicate that there is a dearth of warm DZ MWDs. This may indicate that magnetic fields in WDs with first or second generation planetary systems could be created a few Gigayears after the formation of the $\mathrm{WD}^{54}$. The problem with this scenario is that the incidence of large gaseous planets with short enough orbital periods to cause a merger and thus field production in the WD, may be far too low to explain the high percentage of magnetism among DZ WDs.

Another unique object that cannot be forgotten when discussing planets around WDs is GD 356 with a field strength of $\sim 13$ MG. GD 356 exhibits Zeeman triplets of $\mathrm{H} \alpha$ and $\mathrm{H} \beta$ in emission. The modelling of the spectral features of this star has shown that the emission lines are formed in a region covering a tenth of the stellar surface where the stellar atmosphere has an inverted temperature distribution 57 . Radio observations have failed to provide arguments in support of a magnetic active corona and IR observations to search for a low-mass stellar companion have also given no results 58 . In order to explain these observations, it has been argued that this MWD may have a planetary companion in a close orbit that was strippeddown to its iron core $\frac{59}{9}$ during the post-MS evolution. Electrical currents flowing between the MWD and the planet would cause the heating, via ohmic dissipation, of the upper layers of the MWD's atmosphere. The loss of energy due to Ohmic dissipation would then cause the orbit to decay ${ }^{59}$. The model's prediction was that GD 356 should exhibit periodic variations of several hours. Such a variability was indeed detected in $2004^{60}$ and found to be consistent with the modulation of a spot located near the spin axis and covering $\sim 10 \%$ of the stellar surface. This electric current model is also known as "the unipolar inductor model" 61.

Thus, WDs can be effectively used to gain insight on how planets form around Sun-like stars, which are their progenitors. In this context, I draw attention on new research on Earth-like planets around cool WDs ${ }^{62,63}$. The authors theorise about how planets could stay in the habitable zone of a cool WD for as long as 8 Gyr. They also discuss how planets could end up in such a zone and propose that they could form from material orbiting the WD, possibly as a result of binary interaction or merger events 58,64 . This process would be similar to that invoked to explain the presence of planets originating from accretion discs formed after supernova events around neutron stars. 65,66 . These planets are often called "second generation planets". Alternatively, planets may end up orbiting the WD via capture or through migration from outer to inner orbits 67 . This kind of formation scenario would be applicable to any planet found in a close orbit around a WD star.

\section{Acknowledgments}

The author wishes to thank her collaborators, Dayal Wickramasinghe, Chris Tout, Stephane Vennes and Adela Kawka for many long and stimulating discussions. 


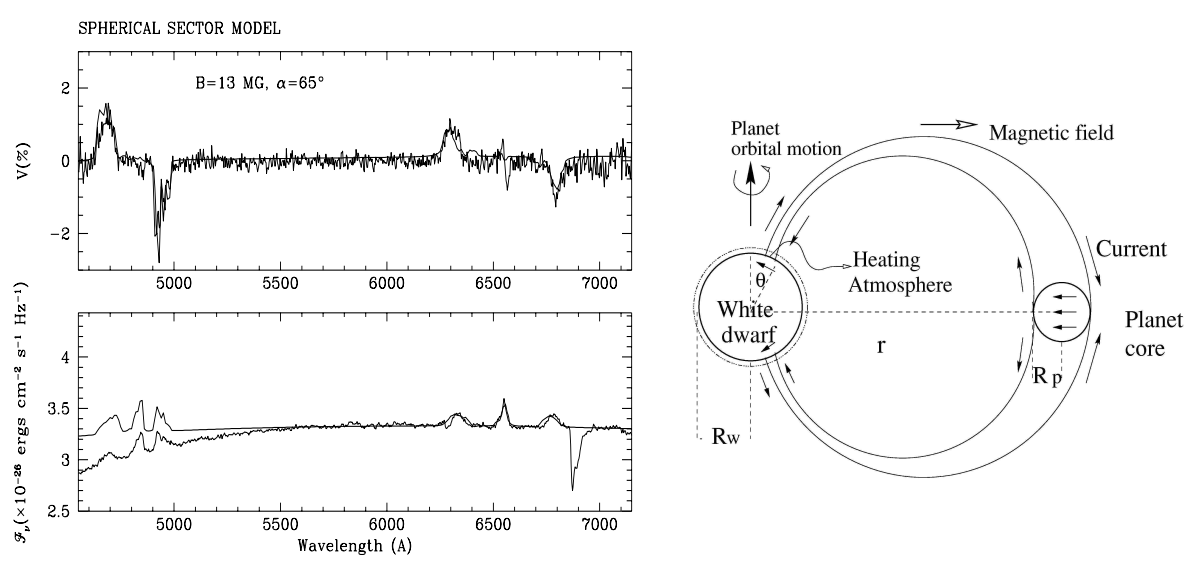

Fig. 4. Left panel: Observed intensity (lower panel) and polarisation (upper panel) spectra of GD 356 showing its characteristic emission features. The calculated spectra are superimposed to the data. Right panel: Electric currents are generated by the highly conductive iron core of a planet around the MWD. The electric circuit that is setup heats the MWD's atmosphere in a region near its magnetic pole causing temperature inversion and the formation of emission lines.

\section{References}

1. G. P. Kuiper, Publ. Astron. Soc. Pac. 46, p. 287 (1934).

2. D. T. Wickramasinghe and L. Ferrario, Ap. J. 327, 222 (1988).

3. L. Ferrario, D. de Martino and B. T. Gänsicke, Space Sci. Rev. 191, 111 (2015).

4. L. Ferrario, D. T. Wickramasinghe, J. Bailey, J. H. Hough and I. R. Tuohy, MNRAS 256, 252 (1992).

5. L. Ferrario, J. Bailey and D. T. Wickramasinghe, MNRAS 262, 285 (1993).

6. L. Ferrario, D. T. Wickramasinghe and A. R. King, MNRAS 260, 149 (1993).

7. G. D. Schmidt, S. Vennes, D. T. Wickramasinghe and L. Ferrario, MNRAS 328, 203 (2001).

8. L. Ferrario, A. Melatos and J. Zrake, Space Sci. Rev. 191, 77 (2015).

9. D. T. Wickramasinghe and L. Ferrario, MNRAS 356, 1576 (2005).

10. A. Rebassa-Mansergas, C. Agurto-Gangas, M. R. Schreiber, B. T. Gänsicke and D. Koester, MNRAS 433, 3398 (2013).

11. J. B. Holberg, Journal of Physics Conference Series 172, p. 012022 (2009).

12. J. B. Holberg and K. Magargal, in 14th European Workshop on White Dwarfs, eds. D. Koester and S. Moehler, Astronomical Society of the Pacific Conference Series, Vol. 334 (2005).

13. J. Liebert, D. T. Wickramasinghe, G. D. Schmidt, N. M. Silvestri, S. L. Hawley, P. Szkody, L. Ferrario, R. F. Webbink, T. D. Oswalt, J. A. Smith and M. P. Lemagie, AJ 129, 2376 (2005).

14. J. Liebert, L. Ferrario, D. T. Wickramasinghe and P. S. Smith, Ap. J. 804, p. 93 (2015).

15. C. A. Tout, D. T. Wickramasinghe, J. Liebert, L. Ferrario and J. E. Pringle, 
MNRAS 387, 897 (2008).

16. D. T. Wickramasinghe, C. A. Tout and L. Ferrario, MNRAS 437, 675 (2014).

17. J. Liebert, Publ. Astron. Soc. Pac. 100, 1302 (1988).

18. P.-E. Tremblay, H.-G. Ludwig, M. Steffen and B. Freytag, $A \mathscr{E} A$ 559, p. A104 (2013).

19. G. P. Briggs, L. Ferrario, C. A. Tout, D. T. Wickramasinghe and J. R. Hurley, MNRAS 447, 1713 (2015).

20. J. Nordhaus, S. Wellons, D. S. Spiegel, B. D. Metzger and E. G. Blackman, Proceedings of the National Academy of Science 108, 3135 (2011).

21. E. García-Berro, P. Lorén-Aguilar, G. Aznar-Siguán, S. Torres, J. Camacho, L. G. Althaus, A. H. Córsico, B. Külebi and J. Isern, Ap. J. 749, p. 25 (2012).

22. C. A. Tout, D. T. Wickramasinghe and L. Ferrario, MNRAS 355, L13 (2004).

23. L. Ferrario, MNRAS 426, 2500 (2012).

24. B. Rolland and P. Bergeron, in 19th European Workshop on White Dwarfs, eds. P. Dufour, P. Bergeron and G. Fontaine, Astronomical Society of the Pacific Conference Series, Vol. 493 (2015).

25. E. V. Linder, Phys. Rev. D 74, p. 103518 (2006).

26. J. R. Hurley, C. A. Tout, D. T. Wickramasinghe, L. Ferrario and P. D. Kiel, MNRAS 402, 1437 (2010).

27. L. Ferrario and D. Wickramasinghe, MNRAS 375, 1009 (2007).

28. L. Ferrario and D. Wickramasinghe, MNRAS 367, 1323 (2006).

29. L. Ferrario and D. Wickramasinghe, MNRAS 389, L66 (2008).

30. S. Ji, R. T. Fisher, E. García-Berro, P. Tzeferacos, G. Jordan, D. Lee, P. LorénAguilar, P. Cremer and J. Behrends, Ap. J. 773, p. 136 (2013).

31. J. Liebert, P. Bergeron, G. D. Schmidt and R. A. Saffer, Ap. J. 418, p. 426 (1993).

32. E. P. Nelan, AJ 134, 1934 (2007).

33. G. Nelemans, R. Napiwotzki, C. Karl, T. R. Marsh, B. Voss, G. Roelofs, R. G. Izzard, M. Montgomery, T. Reerink, N. Christlieb and D. Reimers, $A \& A 440$, 1087 (2005).

34. A. Kawka and S. Vennes, MNRAS 425, 1394 (2012).

35. A. Kawka, L. Ferrario, S. Vennes, E. Paunzen and D. T. Wickramasinghe, MNRAS 1, 1 (2016).

36. S. Vennes. private communication.

37. C. S. Brinkworth, M. R. Burleigh, K. Lawrie, T. R. Marsh and C. Knigge, Ap. J. 773, p. 47 (2013).

38. G. D. Schmidt, et al. Ap. J. 595, 1101 (2003).

39. A. Gianninas, P. Bergeron and M. T. Ruiz, Ap. J. 743, p. 138 (2011).

40. S. Vennes, G. D. Schmidt, L. Ferrario, D. J. Christian, D. T. Wickramasinghe and A. Kawka, Ap. J. 593, 1040 (2003).

41. J. Farihi, E. E. Becklin and B. Zuckerman, Ap. J. 681, 1470 (2008).

42. K. A. Lawrie, M. R. Burleigh, B. N. Barlow, D. O’Donoghue, M. A. Barstow, 
T. R. Marsh, D. Kilkenny and H. Worters, in 18th European White Dwarf Workshop., Astronomical Society of the Pacific Conference Series Vol. 469 (2013).

43. P. D. Dobbie, R. Baxter, B. Külebi, Q. A. Parker, D. Koester, S. Jordan, N. Lodieu and F. Euchner, MNRAS 421, 202 (2012).

44. P. D. Dobbie, B. Külebi, S. L. Casewell, M. R. Burleigh, Q. A. Parker, R. Baxter, K. A. Lawrie, S. Jordan and D. Koester, MNRAS 428, L16 (2013).

45. J. Girven, B. T. Gänsicke, B. Külebi, D. Steeghs, S. Jordan, T. R. Marsh and D. Koester, MNRAS 404, 159 (2010).

46. S. Vennes, L. Ferrario and D. T. Wickramasinghe, MNRAS 302, L49 (1999).

47. A. Putney, Astrophys. J. Suppl. Ser. 112, 527 (1997).

48. E. P. Nelan, H. E. Bond and G. Schaefer, in 19th European Workshop on White Dwarfs, eds. P. Dufour, P. Bergeron and G. Fontaine, Astronomical Society of the Pacific Conference Series, Vol. 493 (2015).

49. B. Zuckerman, D. Koester, I. N. Reid and M. Hünsch, Ap. J. 596, 477 (2003).

50. A. Kawka and S. Vennes, A\& A 532, p. A7 (2011).

51. A. Kawka and S. Vennes, MNRAS 439, L90 (2014).

52. M. A. Hollands, B. T. Gänsicke and D. Koester, MNRAS 450, 681 (2015).

53. S. O. Kepler, MNRAS 446, 4078 (2015).

54. J. Farihi, P. Dufour, R. Napiwotzki and D. Koester, MNRAS 413, 2559 (2011).

55. J. Farihi, M. A. Barstow, S. Redfield, P. Dufour and N. C. Hambly, MNRAS 404, $2123(2010)$.

56. B. Zuckerman, C. Melis, B. Klein, D. Koester and M. Jura, Ap. J. 722, 725 (2010).

57. L. Ferrario, D. T. Wickramasinghe, J. Liebert, G. D. Schmidt and J. H. Bieging, MNRAS 289, 105 (1997).

58. D. T. Wickramasinghe, J. Farihi, C. A. Tout, L. Ferrario and R. J. Stancliffe, MNRAS 404, 1984 (2010).

59. J. Li, L. Ferrario and D. Wickramasinghe, Astrophys. J. Lett. 503, L151 (1998).

60. C. S. Brinkworth, M. R. Burleigh, G. A. Wynn and T. R. Marsh, MNRAS 348, L33 (2004).

61. A. J. Willes and K. Wu, A $\&$ A 432, 1091 (2005).

62. E. Agol, Astrophys. J. Lett. 731, p. L31 (2011).

63. L. Fossati, S. Bagnulo, C. A. Haswell, M. R. Patel, R. Busuttil, P. M. Kowalski, D. V. Shulyak and M. F. Sterzik, Astrophys. J. Lett. 757, p. L15 (2012).

64. M. Livio, J. E. Pringle and K. Wood, Astrophys. J. Lett. 632, L37 (2005).

65. A. Wolszczan and D. A. Frail, Nature 355, 145 (1992).

66. E. S. Phinney and B. M. S. Hansen, in Planets Around Pulsars, eds. J. A. Phillips, S. E. Thorsett and S. R. Kulkarni, Astronomical Society of the Pacific Conference Series, Vol. 36 (1993).

67. J. H. Debes and S. Sigurdsson, Ap. J. 572, 556 (2002). 